ISSN 0103-8478

\title{
Pré-tratamentos na secagem e reidratação de champignon em fatias
}

\author{
Pretreatments in drying and rehydration of mushrooms slices
}

\section{Hayana Juliani Mimura ${ }^{\mathrm{I}}$ Regina Maria Matos Jorge ${ }^{\mathrm{I}}$ Alvaro Luiz Mathias ${ }^{\mathrm{I}^{*}}$}

RESUMO

O objetivo deste trabalho foi avaliar o efeito de dois pré-tratamentos (pt) na desidratação osmo-convectiva: Pré-tratamento químico a $20^{\circ} \mathrm{C}$ por $5 \mathrm{~min}$ em solução de ácido cítrico, bissulfito de sódio e carbonato de cálcio a 0,1\% cada (pt$A B C)$ e por branqueamento a $80^{\circ} \mathrm{C}$ por $5 \mathrm{~min}$ (pt-B), bem como a combinação pt-ABC seguido de pt-B (amostra: solução $=1: 10 \mathrm{w} / \mathrm{w}$ ). $O$ pt-ABC provocou aumento de massa (13\% em média) entretanto o pt-B perda (-34\% em média). A desidratação osmótica a $20^{\circ} \mathrm{C}$ por 60min em solução de $\mathrm{NaCl}$ a $10 \%$ reduziu a massa de fatias não pré-tratadas em 43\%. Esse nível de redução de massa não pode ser atingido com o uso do pt-ABC, mas melhorou para $49 \%$ para fatias submetidas ao $\mathrm{pt}-\mathrm{B}$. Os pré-tratamentos reduziram o tempo de secagem $\left(70^{\circ} \mathrm{C}\right.$ e com circulação de ar de $\left.18 \mathrm{~m}^{3} \mathrm{~h}^{-1}\right)$ de $150 \mathrm{~min}$ para $60 \mathrm{~min}$ para atingir o nível de atividade de água do produto seco igual ou menor a do produto comercial. As fatias submetidas ao pt-ABC ou do controle apresentaram boa capacidade de reidratação. As fatias que foram submetidas aos dois pré-tratamentos apresentaram coloração mais clara e menos enrugadas após secagem, aparência similar foi observada às do ptABC. O pt-ABC pode ser usado para se obter fatias de champignon seco com aspecto visual e capacidade de reidratação adequados para o desenvolvimento de novos produtos para consumo direto ou que necessite reidratação.

Palavras-chave: Agaricus bisporus, desidratação, pré-tratamento químico, branqueamento.

\section{ABSTRACT}

The objective of this paper was assessments the effect of pre-treatment (pt) in osmo-convective dehydration: Chemical pretreatment at $20^{\circ} \mathrm{C}$ for $5 \mathrm{~min}$ with $0.1 \%$ solution of citric acid, sodium bisulfite and calcium carbonate ( $p t-A B C)$ and bleaching at $80^{\circ} \mathrm{C}$ for $5 \mathrm{~min}(\mathrm{pt}-\mathrm{B})$, as well the combination $\mathrm{pt}-\mathrm{ABC}$ followed by $p t-B$ (sample:solution $=1: 10 \mathrm{w} / \mathrm{w})$. The $\mathrm{pt}-\mathrm{ABC}$ provoked mass increase (13\% on average), while pt-B caused loss $(-34 \%$ on average). Osmotic dehydration at $20^{\circ} \mathrm{C}$ for $60 \mathrm{~min}$ with $10 \% \mathrm{NaCl}$ reduced mass of slices not pretreated at 43\%. This level of mass reduction was not achieved for the pt-ABC, but it was improved for $49 \%$ to slices subjected to $p t-B$. Pretreatments reduced drying time $\left(70^{\circ} \mathrm{C}\right.$ and air circulation $\left.18 \mathrm{~m}^{3} \mathrm{~h}^{-1}\right)$ from $150 \mathrm{~min}$ to $60 \mathrm{~min}$ to achieve the desired $a_{w}$. Water activity of dried product was equal to or less than the commercial product. Slices subjected to pt-ABC or from control group had good rehydration capacity. Slices subjected to combined pt and dried showed lighter color and were less wrinkled. pt-ABC allowed very similar results. The pt-ABC must be used to obtain slices of dry champignon with visual aspect and rehydration capacity suitable for the development of new products for direct consumption or require rehydration.

Key words: Agaricus bisporus, dehydration, chemical pretreatment, blanching.

\section{INTRODUÇÃO}

A desidratação é utilizada para aumentar a conservação de alimentos através da redução do conteúdo de umidade. Essa operação provoca redução da atividade de água, inibição de atividade de enzimas, inibição do crescimento de microrganismos deteriorantes e redução do volume e de massa do produto final; o que facilita a conservação e reduz os custos com transporte e armazenamento (DINCER \& DOST, 1995). Cogumelos, brócolis, cenoura, ervilha, milho, cebola, alho e abobrinha são exemplos de vegetais que podem ser desidratados. Nesta apresentação, esses alimentos podem ser adicionados como componentes de formulação de molhos para salada e sopas instantâneas ou, em alguns casos, ser consumidos diretamente como chips temperados (TULEY, 1996; TORRINGA et al., 2001).

\footnotetext{
'Departamento de Engenharia Química, Universidade Federal do Paraná (UFPR), 81531-980, Curitiba, PR, Brasil. E-mail: mathias@ufpr.br. *Autor para correspondência. 
A remoção de água do tecido vegetal pode ser feita por desidratação osmótica. Nesta operação, o tecido vegetal é exposto a uma solução relativamente rica em solutos iônicos ou moleculares, os quais induzem a saída de água do vegetal. No entanto, somente essa operação não consegue reduzir a umidade a níveis que possa aumentar a vida de prateleira do produto, pois a atividade de água não é reduzida aos níveis restritivos ao desenvolvimento microbiano (MALTINI et al., 2003). Assim, operações complementares são necessárias; por exemplo, a secagem convectiva (TORRINGA et al., 2001). $\mathrm{O}$ agente de desidratação osmótica mais utilizada para produtos salgados é o cloreto de sódio; como descrito para tomate (BARONI, 2004), abóbora (BORIN et al., 2008) e cogumelo (TORRINGA et al., 2001; KUROZAWA et al., 2005). O consumo direto do produto seco nem sempre é única forma de demanda. Assim, é conveniente que ele também possa ser reidratado para ser utilizado de modo similar ao alimento fresco. O objetivo desta operação é de recuperar suas propriedades de quando era um produto fresco ao entrar em contato com água e/ou vapor de água. No entanto, as alterações estruturais causadas pela secagem convectiva impedem que a reidratação de produtos secos seja completa (LEE et al., 2006).

$\mathrm{O}$ uso de secagem de produtos fatiados é muito comum, mas favorece a alteração do seu aspecto visual e sua cor (RAMOS et al., 2003). No caso do champignon, o escurecimento é atribuído à ação de enzimas, como a tirosinase e a catecolase; sendo que suas inativações podem ser realizadas com uso de calor, contato com sulfito, ou redução do pH (BURTON, 1980; MODA et al., 2005). Logo, a imersão de suas fatias em soluções com sulfitos ou metabissulfitos, associado ou não a ácido cítrico, controla efetivamente o escurecimento enzimático; como relatado para batata, cenoura e maçã (DAMODARAN et al., 2010). A imersão em solução de carbonato de cálcio é outra operação para melhorar as propriedades finais deste tipo de alimento. Este sal atua como agente de manutenção ou melhoramento de propriedades, como a textura (BRASIL, 1999). O branqueamento é outro tratamento descrito para estabilizar a cor original do produto final. $\mathrm{O}$ alimento fragmentado deve ser imerso em água aquecida para inibir a ação enzimática e o desenvolvimento de microrganismo (FELLOWS, 2000). Assim, operações de pré-tratamento $(\mathrm{pt})$ podem anteceder a operação de desidratação de um produto alimentar com o objetivo de melhorar suas propriedades. Neste estudo, os efeitos do pré-tratamento químico com uma solução aquosa contendo ácido cítrico, bissulfito de sódio e carbonato de cálcio (denominado de $\mathrm{ABC}$ ) e/ou por branqueamento (denominado de B) com água aquecida foram avaliados com o propósito de melhorar as propriedades visuais (textura superficial e cor) de fatias de champignon secos com uso de desidratação osmo-convectiva. Por último, a reidratação do produto seco também foi estudada para determinar o efeito dos diferentes tipos de prétratamentos e, consequentemente, o melhor grau de incorporação de água.

\section{MATERIAL E MÉTODOS}

O Champignon de Paris (Agaricus bisporus) in natura foi adquirido no Mercado Municipal de Curitiba, Paraná, sempre do mesmo produtor, localizado no Município de Colombo, Paraná. Quatro rotas que poderiam anteceder a desidratação osmo-convectiva foram realizadas (Figura 1): O controle (a), o branqueamento puro (b: pt-B), o pré-tratamento químico puro (c: pt-ABC) ou a combinação destes (d: ABC-B); na ordem, químico e depois branqueamento. Todos em condição estática. Champignons de Paris in natura apresentavam umidade de $93,0 \% \pm 0,06 \%$. Os espécimes com cerca de $3 \mathrm{~cm}$ de diâmetro foram lavados em água corrente, drenados e sua água superficial foi removida com papel absorvente (esta última operação foi denominada de "secagem superficial"). Suas fatias longitudinais com espessura entre 4 e $5 \mathrm{~mm}$ foram produzidas em cortador manual. Imediatamente, cerca de $20 \mathrm{~g}$ foram submetidos à desidratação osmótica e secagem convectiva (a: controle). Alternativamente, as fatias foram imersas em água destilada previamente estabilizadas $80^{\circ} \mathrm{C}$ e permaneceram no banho por $5 \mathrm{~min}$ (b: pt-B). Outra porção de fatias foi imersa em $200 \mathrm{~g}$ de solução de ácido cítrico $(0,1 \%)$, de bissulfito de sódio $(0,1 \%)$ e de carbonato de cálcio $(0,1 \%)$ a $20^{\circ} \mathrm{C}$ por $5 \mathrm{~min}$ em béqueres de $250 \mathrm{~mL}$ de forma alta (c: pt-ABC). Por último, uma segunda porção, também submetida a este tratamento, foi submetida ao processo de branqueamento (d: ABC-B). Antes e no final do pré-tratamento, as fatias foram drenadas, submetidas a "secagem superficial" e pesadas. Logo, a variação de massa em base úmida foi calculada para cada operação.

A desidratação osmótica (DO) foi realizada sob agitação (70rpm) para fatias submetidas a cada uma das quatro rotas. A DO foi realizada com a imersão de cerca de $20 \mathrm{~g}$ de fatias em aproximadamente $200 \mathrm{~g}$ de solução de cloreto 


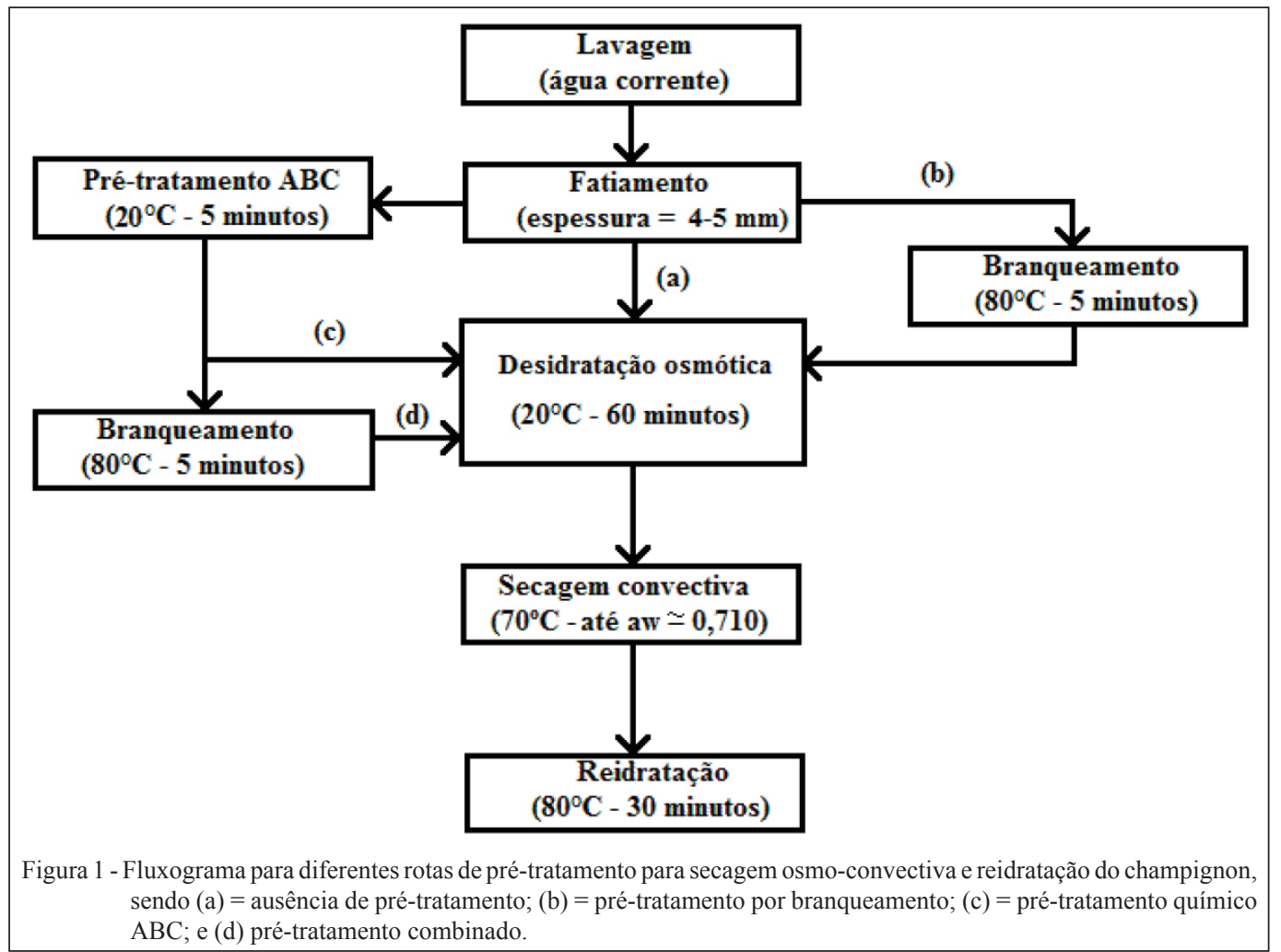

de sódio a $10 \%$ contida em béqueres de $250 \mathrm{~mL}$ a temperatura ambiente (cerca de $20^{\circ} \mathrm{C}$ ) por $60 \mathrm{~min}$. A relação entre a massa de fatias e volume de solução de DO foi constante $(1: 10)$. As fatias de champignons para diferentes tempos de DO $(0,20,40$ ou 60min) foram lavadas rapidamente com água destilada, drenadas, "secas superficialmente" e pesadas. Após desidratação osmótica, aproximadamente 30 fatias foram colocadas em telas de alumínio e secas em secador convectivo do tipo bandejas (FABBE modelo 170 , Itu, SP, Brasil) a $70^{\circ} \mathrm{C}$ e com circulação de ar de $18 \mathrm{~m}^{3} \mathrm{~h}^{-1}$. Três dessas fatias foram usadas para determinar a atividade de água $\left(\mathrm{a}_{\mathrm{w}}\right)$. As amostras foram retiradas em intervalos de 0,15 , $30,60,90,120,150 \mathrm{~min}$. O tempo de secagem que não apresentou alteração importante de massa em relação ao seu sucessor e que atingiu atividade de água $\left(\mathrm{a}_{\mathrm{w}}\right)$ igual ou inferior a 0,71 foi considerado como tempo ideal de secagem. A atividade de água foi determinada com a utilização de um higrômetro (Aqualab Decagon Devices, modelo Série 3TE, Pullman, WA, EUA) à temperatura climatizada a $25^{\circ} \mathrm{C}$ ao longo de todo o processo de secagem. A $\mathrm{a}_{\mathrm{w}}$ de 0,710 foi adotada como referência baseado no valor observado em produto similar (cogumelo Funghi sp.) comercializado em Curitiba, PR, Brasil; o qual é consumido na forma reidratada.
Uma porção com cerca de $3 \mathrm{~g}$ destas fatias secas foram reidratados em $200 \mathrm{~g}$ de água destilada à temperatura de $80^{\circ} \mathrm{C}$ por $30 \mathrm{~min}$. A massa inicial e final foi determinada, de modo similar aos prétratamentos, nos tempos $0,10,20$ e 30 minutos para avaliar aumento em porcentagem da massa inicial. A imagem fotográfica para avaliação sensorial da cor e da "textura visual" foi feita com uso de uma máquina fotográfica Olympus (modelo Stylus 790 sw) posicionada paralelamente à superfície das fatias de champignon e a mesma distância para todas as tomadas. A exposição selecionada foi automática. A iluminação usada foi luz natural e todas as tomadas foram realizadas no mesmo dia e hora para as diferentes amostras. Os valores de variação de massa resultante dos pré-tratamentos e da desidratação osmótica apresentados são a média de triplicatas, enquanto o desvio-padrão é apresentado como medida de dispersão.

\section{RESULTADOS E DISCUSSÃO}

As fatias de champignon foram submetidas ou não a diferentes pré-tratamentos $(\mathrm{pt})$ (Figura 1). O pré-tratamento químico provocou aumento de massa (Tabela 1), sendo 13\% (c: pt$\mathrm{ABC})$ e $14 \%$ (d: ABC-B), enquanto que o pré-

Ciência Rural, v.44, n.4, abr, 2014. 
tratamento por branqueamento provocou grande redução de massa, sendo $-36 \%$ (b: pt-B) e $-32 \%$ (d: ABC-B). Logo, pt-B provoca forte desidratação. Isso pode ser atribuído à possível perda de água, mas também de sólidos do alimento, o que é um fato extremamente negativo para a qualidade final de um produto alimentício. No entanto, o pt$\mathrm{ABC}$ tem esse fenômeno negligenciável e produz incorporação de sólidos do meio aquoso nas fatias (ZOU et al., 2013). A desidratação osmótica (DO) acarretou uma importante redução de massa para as fatias não branqueadas (Tabela 1); sendo $-43 \%$ para o controle (a) e $-41 \%$ para pt-ABC (c), o que reforça seu uso antes de outros processos complementares de secagem (ZOU et al., 2013). Por outro lado, a DO foi menos eficaz na redução de massa para rotas com branqueamento, pois reduziu apenas $-13 \%$ com pt-B (b) e $-7 \%$ com pt combinado (d: ABC-B). Ainda, a perda de massa nas amostras controle $(-43 \%)$ e pt-B (-49\%) causada pela desidratação osmótica foi maior em relação aos resultados obtidos nas amostras prétratadas quimicamente pt-ABC (-28\%) e ABC-B (-24\%); conforme coluna $1^{\circ} \mathrm{pt}-\mathrm{DO}$ da tabela 1 . Observando apenas esses aspectos, seria plausível afirmar que a DO e o pt-ABC são dispensáveis, pois se necessita duas operações e maiores custos para aquisição de aditivos químicos para atingir mesma redução de massa. Esse fato talvez possa justificar diversos processos de secagem que não utilizam a desidratação osmótica prévia (PARK et al., 2011). No entanto, tem sido relatado que a presença de sais em soluções de imersão do alimento fatiado reprimi a perda de seus componentes sólidos (PARK et al., 2011; ZOU et al., 2013). A avaliação univariada da perda de massa durante a DO revelou que é função do tempo (Figura 2), pois se estabilizou a $40 \mathrm{~min}$ para ambas as rotas sem pt-B (a: controle e c: pt$\mathrm{ABC}$ ). As rotas com branqueamento, b (pt-B) ou d (ABC-B), tiveram uma importante variação de massa apenas até 20min (Figura 2).
A secagem das amostras submetidas ou não a um ou ambos pré-tratamentos foi realizada logo após a DO (Figura 1). As fatias sem prétratamento (a: controle) necessitaram 150min de secagem (Tabela 1), enquanto que as fatias de champignon submetidas a um ou ambos prétratamentos precisaram apenas $60 \mathrm{~min}$ de secagem. Este é um importante aspecto de decisão de processo industrial, pois revela que o pré-tratamento economiza energia de processo de secagem. É uma economia de $60 \%$ em relação ao controle (a). A atividade de água $\left(\mathrm{a}_{\mathrm{w}}\right)$ ao final da secagem foi medida (Tabela 1), pois é a propriedade que reflete a qualidade e estabilidade de um alimento. Valores entre 0,60 e 0,65 são comuns para frutas secas (MALTINI et al., 2003). Não foram encontrados dados específicos para cogumelos; logo, $\mathrm{a}_{\mathrm{w}}$ foi medida para cogumelo seco comercializado na região de estudo $(0,710)$ como base de comparação. As fatias de champignon secas sem pré-tratamento (a: controle $\rightarrow \mathrm{a}_{\mathrm{w}}=0,63$ ) ou com tratamento (b: pt-B $\rightarrow \mathrm{a}_{\mathrm{w}}=0,71$; c: pt-ABC $\rightarrow \mathrm{a}_{\mathrm{w}}=0,61, \mathrm{~d}:$ ABC-B $\rightarrow$ $\left.a_{w}=0,68\right)$ apresentaram valores iguais ou inferiores ao produto comercial, o que sugere que possa ser igualmente conservado (MALTINI et al., 2003).

$\mathrm{O}$ aspecto visual pode afetar drasticamente a aceitação de um produto seco. É o que foi relatado nos Estados Unidos para a pera desidratada com uso de secador convectivo. O produto final fica mais claro do que o tradicionalmente obtido pela secagem ao sol e, portanto, não teve boa aceitação pelo consumidor. (PARK et al., 2001). No Brasil, o Champignon de Paris é tradicionalmente consumido com coloração clara e espera-se que este seja uma propriedade desejada no produto reidratado. $\mathrm{O}$ aspecto visual da textura superficial e da coloração das fatias champignons (Figura 3) sem pré-tratamento (a: controle) produziu um cogumelo muito escuro e com textura levemente enrugada, enquanto o que sofreu pt-B (b) ficou relativamente claro e com textura

Tabela 1 - Variação de massa entre as operações de pré-tratamento ou associado à desidratação osmótica, tempo de secagem a níveis de armazenamento e atividade de água do chips.

\begin{tabular}{|c|c|c|c|c|c|c|}
\hline Rotas* & $\mathrm{ABC}(\mathrm{dp})(\%)$ & B (dp) (\%) & DO (dp) $(\%)$ & $1^{\circ} \mathrm{PT}-\mathrm{DO}^{\#}(\%)$ & $\mathrm{TS}$ (min) & $a_{w}(d p)$ \\
\hline $\mathrm{a}$ & - & - & $-43,35( \pm 2,13)$ & $-43,35$ & 150 & $0,629( \pm 0,053)$ \\
\hline $\mathrm{b}$ & - & $-35,95(1,32)$ & $-13,09( \pm 2,03)$ & $-49,04$ & 60 & $0,712( \pm 0,025)$ \\
\hline $\mathrm{c}$ & $+13,17( \pm 1,10)$ & - & $-40,86( \pm 2,15)$ & $-27,69$ & 60 & $0,613( \pm 0,045)$ \\
\hline $\mathrm{d}$ & $+14,04( \pm 1,10)$ & $-31,57( \pm 1,23)$ & $-6,77(1,68)$ & $-24,30$ & 60 & $0,677( \pm 0,050)$ \\
\hline
\end{tabular}

*Onde: a: controle, b: somente pré-tratamento por branqueamento (B), c: somente pré-tratamento químico (ABC), d: pré-tratamento combinado $(\mathrm{ABC}-\mathrm{B}), 1^{\mathrm{o}} \mathrm{PT}=$ primeiro pré-tratamento, DO: desidratação osmótica, TS: tempo de secagem convectiva; $\mathrm{a}_{\mathrm{w}}$ : atividade da água a $25^{\circ} \mathrm{C}$ depois da secagem convectiva. \# Variação de massa entre o início da primeira operação e o final da DO. dp $=$ desvio padrão de triplicatas. 


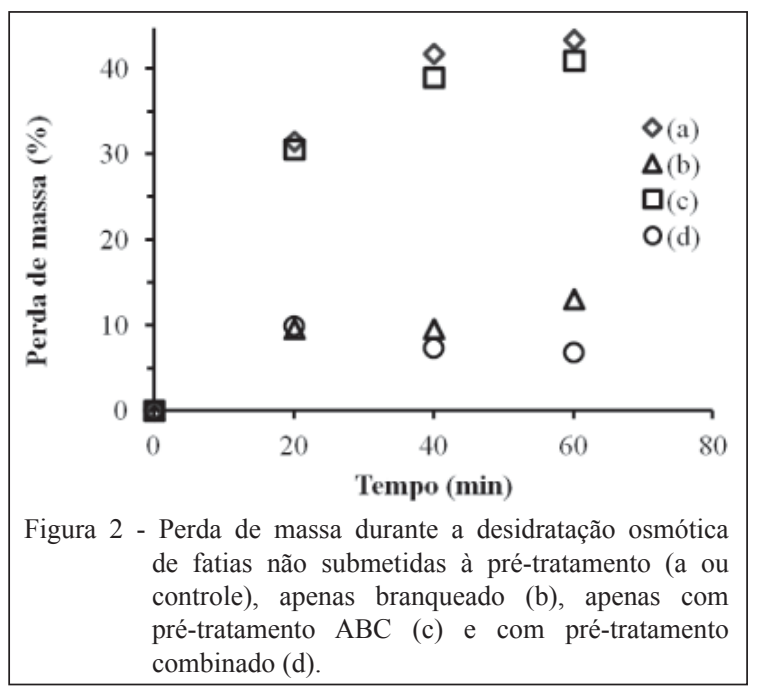

enrugada. O produto que sofreu pt-ABC (c) era claro e com textura agradável. $\mathrm{O}$ pt-B e o pt-ABC reduziram a ação deletéria das enzimas endógena do cogumelo, seja por inativação com uso de temperatura elevada (FELLOWS, 2000) ou com uso de bissulfito associado com ácido cítrico (BURTON, 1980; MODA et al., 2005). O produto que sofreu ambos pré-tratamentos (d) era mais agradável (o mais claro e o menos enrugado). $\mathrm{O}$ aspecto do cogumelo previamente prétratado com solução de sais e depois desidratado por osmo-convecção confirma que a textura final de um produto pode ser melhorada com a imersão em solução de carbonato de cálcio (BRASIL, 1999). Ainda, a associação com o branqueamento sugere que pode ter havido uma desidratação superficial menos severa, o que torna sua textura menos enrugada ainda, se for removido parte dos sais impregnados durante o pt-ABC. De modo geral, as rotas com pt ABC (c: pt$\mathrm{ABC}$ e d: $\mathrm{ABC}-\mathrm{B})$ são convenientes.
A reidratação máxima ocorreu praticamente em 10min (Figura 4). A taxa e nível máximo de reidratação das fatias obtidas sem prétratamento (a) foi um pouco maior do que para com pt-ABC (c). As rotas com branqueamento (b e d) apresentaram os piores resultados. Assim, o pt-B é negativo para reidratação do produto, enquanto o pt$\mathrm{ABC}$ não interfere de modo decisivo nessa operação. $\mathrm{O}$ aumento de massa após reidratação durante $30 \mathrm{~min}$ foi de $140,06 \%$ para rota sem pré-tratamento (a: controle), 60,47\% com apenas branqueamento (b), $109,85 \%$ com apenas pré-tratamento químico (c) e $49,78 \%$ para ambos pré-tratamentos (d). Esses valores são inferiores aos valores iniciais devido às possíveis alterações estruturais (LEE et al., 2006). Desta forma, o produto com pt-ABC (c) é mais conveniente para produzir fatias de champignon secas em alimentos que necessitem rápida hidratação, como sopas instantâneas, devido a sua boa aparência e boa capacidade de reidratação; mas também com coloração clara.

\section{CONCLUSÃO}

O cogumelo seco produzido sem prétratamento apresentou o pior aspecto visual para textura superficial e cor, bem como necessita de um maior tempo de secagem em relação às amostras pré-tratadas. O cogumelo seco obtido com uso de apenas pré-tratamento químico apresentou aspecto visual agradável e facilidade de reidratação conveniente para seu uso. O prétratamento $\mathrm{ABC}$ (ácido cítrico a 0,1\%, de bissulfito de sódio a $0,1 \%$ e de carbonato de cálcio a $0,1 \%$ a $20^{\circ} \mathrm{C}$ por $5 \mathrm{~min}$, champignon in natura:solução $1: 10$ ),

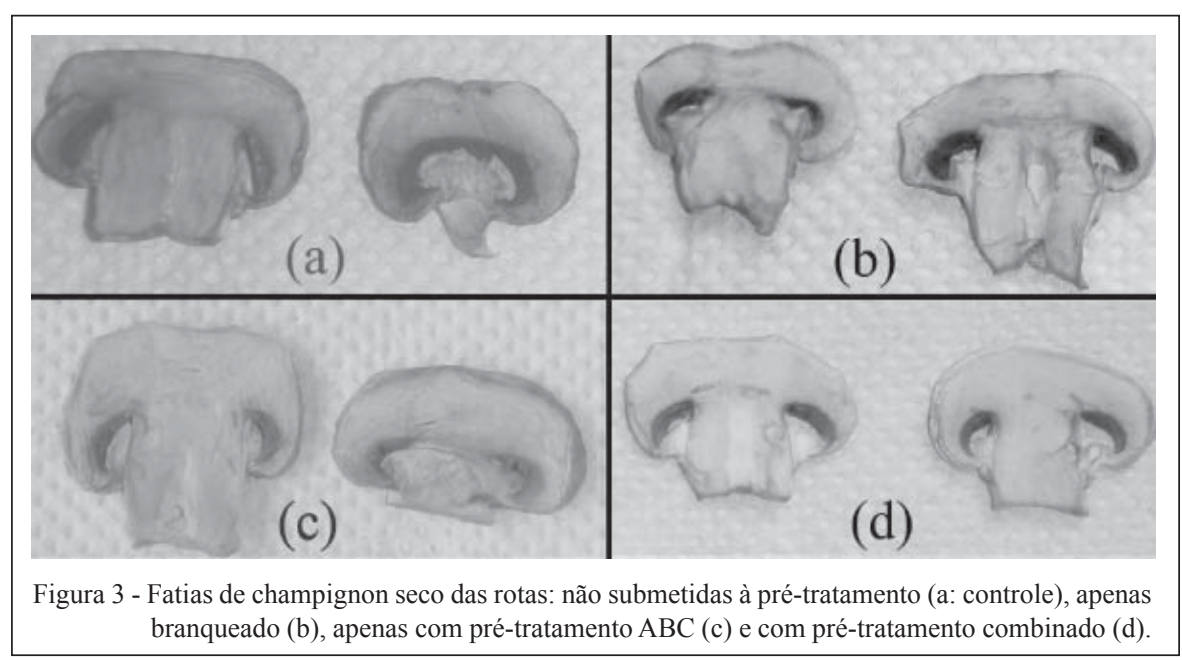

Ciência Rural, v.44, n.4, abr, 2014. 


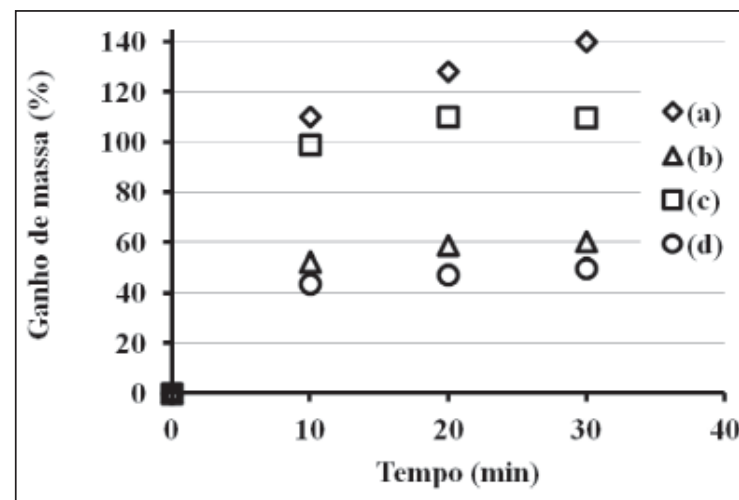

Figura 4 - Ganho de massa dos champignons secos durante a reidratação para fatias de champignon seco das rotas: não submetidas à pré-tratamento (a: controle), apenas branqueado (b), apenas com pré-tratamento $\mathrm{ABC}(\mathrm{c})$ e com pré-tratamento combinado (d).

seguido de desidratação osmótica $\left(\mathrm{NaCl}\right.$ a $10 \%$ a $20^{\circ} \mathrm{C}$ por $40 \mathrm{~min}$, champignon pré-tratado:solução $1: 10$ ) e secagem $\left(70^{\circ} \mathrm{C}\right.$ por $60 \mathrm{~min}$ com circulação de ar de $18 \mathrm{~m}^{3} \mathrm{~h}^{-1}$ ) pode produzir champignon em fatias com a boa aparência e grande capacidade de reidratação.

\section{AGRADECIMENTOS}

Os autores agradecem o apoio financeiro da Universidade Federal do Paraná (UFPR), do Conselho Nacional de Desenvolvimento Científico e Tecnológico (CNPq) e da Coordenação de Aperfeiçoamento Pessoal de Nível Superior (CAPES - REUNI) pelo apoio financeiro e bolsas de estudo.

\section{REFERÊNCIAS}

BARONI A.F. Propriedades mecânicas, termodinâmicas e de estado de tomate submetido à desidratação osmótica e secagem. 2004. 245f. Tese (Doutorado em Engenharia de Alimentos) Faculdade de Engenharia de Alimentos, Universidade Estadual de Campinas (UNICAMP), SP. Disponível em: <http://www.fea. unicamp.br/alimentarium/ver_documento. php?did=440>.Acesso em: 20 nov. 2008.

BORIN, I. et al. Efeito do pré-tratamento osmótico com sacarose e cloreto de sódio sobre a secagem convectiva de abóbora. Ciência e Tecnologia de Alimentos, v.28, n.1, p.39-50, 2008. Disponível em: $<$ http://www.scielo.br/pdf/cta/v28n1/07.pdf>.Acesso em: 03 dez. 2010.

BRASIL. Resolução - RDC n.386, de 05 de agosto de 1999. Regulamento técnico sobre aditivos utilizados segundo as boas práticas de fabricação e suas funções. Disponível em: <http:// portal.anvisa.gov.br/wps/wcm/connect/0556e3004745787485bdd 53fbc4c6735/RESOLUCAO_386_1999.pdf?MOD=AJPERES $>$. Acesso em: 12 jun. 2011.

BURTON, K.S. The effects of pre- and post-harvest development on mushroom tyrosinase. Journal of Horticultural Science, v.63, n.2, p.255-260, 1980. Disponível em: <http://www.jhortscib.org/ Vol63/63_2/11.htm>.Acesso em: 12 jun. 2011.

DAMODARAN, S. et al. Química de alimentos de fennema. 4.ed. Porto Alegre: Artmed, 2010. 900p.
DINCER, I.; DOST, S. An analytical model for moisture diffusion in solid objects during drying. Drying Technology, v.13, p.425435, 1995. Disponível em: <http://www.tandfonline.com/doi/ abs/10.1080/07373939508916962\#.Up73pNKsh8E >.Acesso em: 12 jun. 2011. doi:10.1080/07373939508916962.

FELLOWS, P.J. Food process technology - Principles e practice. 2.ed. São Paulo: CRC, 2000. 575p.

KUROZAWA, L.E et al. Obtenção de isotermas de dessorção de cogumelo in natura e desidratado osmoticamente. Ciência e Tecnologia de Alimentos, v.25, n.4, p.828-834, 2005. Disponível em: <http://www.scielo.br/pdf/cta/v25n4/27659. pdf $>$.Acesso em: 12 jun. 2011. http://dx.doi.org/10.1590/S010120612005000400033.

LEE, K.T. et al. The mathematical modeling of the rehydration characteristics of fruits. Journal of Food Engineering, v.72, p.16-23, 2006. Disponível em: <http://ac.els-cdn.com/ S0260877404005874/1-s2.0-S0260877404005874-main.pdf? tid=9195620e-5cd1-11e3-b75c-00000aacb361\&acdnat $=138615 \overline{4}$ 271_5131880f93961a9e9901281ac8a3fc70>. Acesso em: 12 jun. 2011. doi:10.1016/j.jfoodeng.2004.11.014.

MALTINI, E. et al. Water activity and the preservation of plant foods. Food Chemistry, v.82, p.79-86, 2003. Disponível em: <http://depa. fquim.unam.mx/amyd/archivero/27TVAwTg_14243.pdf $>$.Acesso em: 12 jun. 2011. doi:10.1016/S0308-8146(02)00581-2.

MODA, E.M. et al. Uso de peróxido de hidrogênio e ácido cítrico na conservação de cogumelos Pleurotus sajor-caju in natura. Ciência e Tecnologia de Alimentos, v.25, n.2, p.291-296, 2005. Disponível em: $<$ http://www.scielo.br/scielo.php?script=sci arttext\&pid $=$ S0101-20612005000200019>.Acesso em: 12 jun. 2011. http://dx.doi.org/10.1590/S0101-20612005000200019.

PARK, K.J. et al. Estudo de secagem de pêra bartlett (Pyrus sp.) em fatias. Ciências e Tecnologia de Alimentos, v.21, n.3, p.288-292, 2001. Disponível em: $<$ http://www.scielo.br/scielo.php?script $=$ sci arttext\&pid $=$ S0101-20612001000300007 $>$. Acesso em: 12 jun. 2011. http://dx.doi.org/10.1590/S0101-20612001000300007.

RAMOS, I.N. et al. Structural changes during air drying of fruits and vegetables. Food Science and Technology International, v.9, n.3, p.201-206, 2003. Disponível em: <http://fst.sagepub. com/content/9/3/201.full.pdf + html $>$.Acesso em: 12 jun. 2011. doi: $10.1177 / 1082013030335522$.

TULEY, L. Swell time for dehydrated vegetables. International Food Ingredients, v.4, p.23-27, 1996.

TORRINGA E. et al. Osmotic dehydration as a pre-treatment before combined microwave-hot-air drying of mushrooms. Journal of Food Engineering, v.49, p.185-191, 2001. Disponível em: <http://ac.els-cdn.com/S0260877400002120/1s 2.0-S 0260877400002120 -main.pdf?_tid=99decd4e$5 \mathrm{ccf}-11 \mathrm{e} 3-9 \mathrm{ae} 2-00000 \mathrm{aab} 0 \mathrm{f} 01$ \&acdnat $=1386153426$ ea597d695543c245f20354a153f0fc0f $>$.Acesso em: 12 jun. 2011. doi:10.1016/S0260-8774(00)00212-0.

ZOU, K et al. Effect of osmotic pretreatment on quality of mango chips by explosion puffing drying. Food Science and Technology, v.51, p.253-259, 2013. Disponível em: <http://www.sciencedirect. com/science/article/pii/S0023643812004537>.Acesso em: 12 jun. 2011. http://dx.doi.org/10.1016/j.lwt.2012.11.005. 